\title{
Comment on the Paper Entitled "A New Cumulative Fatigue Damage Rule Based on Dynamic Residual S-N Curve and Material Memory Concept" by Peng Z., Huang H., Zhou J. and Li Y. Published in Metals (2018; 8 (6): 456)
}

\author{
Kris Hectors ${ }^{1,2, *(\mathbb{D})}$ and Wim De Waele ${ }^{1}$ (D) \\ 1 Department of EMSME, Laboratory Soete, Faculty of Engineering and Architecture, Ghent University, \\ Technologiepark 46, BE-9052 Zwijnaarde, Belgium; Wim.DeWaele@UGent.be \\ 2 SIM vzw, Technologiepark 48, BE-9052 Zwijnaarde, Belgium \\ * Correspondence: kris.hectors@ugent.be
}

Received: 18 October 2019; Accepted: 22 October 2019; Published: 19 December 2019

\section{Dear Editors,}

We are writing to draw attention to what we consider to be a consequential error in the research article entitled "A new cumulative fatigue damage rule based on dynamic residual S-N curve and material memory concept" by Peng Z., Huang H., Zhou J. and Li Y. published in Metals (2018; 8 (6): 456).

On page 9 of 17, the authors write that the total fatigue life can be calculated by their Equation (34), i.e., $N_{\text {pre }}=\sum_{i=1}^{k} n_{i} / \sum_{i=1}^{k} D_{i}$. This formula states that the lifetime $\left(N_{\text {pre }}\right)$ of a component subjected to a sequence of load blocks can be predicted by the ratio of the total number of applied cycles $\left(\sum_{i=1}^{k} n_{i}\right)$ and the corresponding accumulated damage $\left(\sum_{i=1}^{k} D_{i}\right)$. Equation (34) implicitly assumes that the number of cycles of each load block can be scaled with the same scalar up to failure, corresponding to $\sum D_{i}=1$ and $\sum n_{i}=N_{\text {pre }}$. This is however only true if the damage accumulation is described as a linear function of cycle ratios. The use of Equation (34) for non-linear damage accumulation models is inherently wrong which can easily be shown by using a two-level load block sequence as an example:

- $\quad$ Piece-wise linear damage model, Kwofie's model

$$
N_{\text {pre }}=\frac{\sum n_{i}}{\sum D_{i}}=\frac{n_{1}+n_{2}}{\frac{n_{1}}{N_{1}}+\frac{n_{2}}{N_{2}} \frac{\ln \left(N_{2}\right)}{\ln (N 1)}}=\frac{C n_{1}+C n_{2}}{C \frac{n_{1}}{N_{1}}+C \frac{n_{2}}{N_{2}} \frac{\ln \left(N_{2}\right)}{\ln (N 1)}}
$$

- $\quad$ Non-linear damage model, model proposed by Peng et al.

$$
N_{\text {pre }}=\frac{\sum n_{i}}{\sum D_{i}}=\frac{n_{1}+n_{2}}{\frac{n_{1}}{N_{1}}+\frac{n_{2}}{N_{2}}\left(\frac{N_{1}}{N_{2}}\right)^{\frac{e^{-\frac{n_{1}}{N 1}}-e^{-1}}{1-e^{-1}}}} \neq \frac{C n_{1}+C n_{2}}{C \frac{n_{1}}{N_{1}}+C \frac{n_{2}}{N_{2}}\left(\frac{N_{1}}{N_{2}}\right)^{\frac{e^{-C} \frac{n_{1}}{N 1}-e^{-1}}{1-e^{-1}}}}
$$

For Equation (1) an unscaled load history results in the same predicted lifetime as when using a load history where the number of cycles of each block is scaled with a scalar $C$, owing to the linearity of the damage model. In the case of a non-linear damage model this is not true anymore as illustrated by Equation (2). The power in the denominator of Equation (2) is called the decay coefficient and is defined in Equation (7) in the paper of Peng et al. Since this coefficient is a function of $n_{1}$, linear scaling of the load block cycles $\sum n_{i}$ (numerator of Equation (2)) results in a non-linear scaling of the accumulated damage $\sum D_{i}$ (denominator of Equation (2)). This means that Equation (34) produces erroneous $N_{\text {pre }}$ 
for the proposed non-linear damage model of Peng et al. Therefore part of the results reported in the paper of Peng et al., more specifically in the Tables $2-4,6,8$ and in Figures $3-5$ are wrong.

$$
\alpha_{1}=\frac{e^{-\frac{n_{1}}{N 1}}-e^{-1}}{1-e^{-1}}
$$

To illustrate the significance of this error, Table 1 is included at the back of this letter. Row (a) of each load scenario (e.g., high-low) shows the lifetime prediction as calculated by Peng et al. Row (b) shows the accumulated damage when the number of applied cycles is equal to $N_{\text {pre }}$. Evidently, the damage sum should be equal to unity in the second case but as shown $\sum D_{i} \neq 1$. Finally, row (c) shows the lifetime prediction based on a correct implementation of the damage model of Peng et al. i.e., the number of cycles corresponding to $\sum D_{i}=1$ is calculated. An error of approximately 5000 cycles is demonstrated; for multi-level block loading this error could become even more significant.

Table 1. Predicted lifetime calculated by Peng et al. for their non-linear damage model and two block loading sequences (a), with the corresponding damage (b) and the corrected lifetime prediction (c).

\begin{tabular}{|c|c|c|c|c|c|c|c|}
\hline \multicolumn{8}{|c|}{ High-Low Loading Sequence $\sigma_{1}=485 \mathrm{MPa}-\sigma_{2}=400 \mathrm{MPa}$} \\
\hline & $n_{1} / n_{2}$ & $n 1$ & $n 2$ & $\sum n_{i}$ & $\alpha_{1}$ & $\sum D_{i}$ & $N_{\text {pre }}$ \\
\hline (a) $N_{\text {pre }}$ Peng et al. & 0.268 & 13,749 & 51,304 & 65,053 & 0.6501 & 0.7450 & 87,314 \\
\hline (b) Corresponding damage & 0.268 & 18,454 & 68,860 & 87,314 & 0.5491 & 1.0687 & \\
\hline (c) Correct $N_{\text {pre }}$ & 0.268 & 17,497 & 65,290 & 82,787 & 0.5689 & 1.0000 & 82,786 \\
\hline \multicolumn{8}{|c|}{ Low-High Loading Sequence $\sigma_{1}=400 \mathrm{MPa}-\sigma_{2}=485 \mathrm{MPa}$} \\
\hline (a) $N_{\text {pre }}$ Peng et al. & 2.341 & 109,310 & 46,693 & 156,003 & 0.1653 & 1.1264 & 138,502 \\
\hline (b) Corresponding damage & 2.341 & 97,047 & 41,455 & 138,502 & 0.2309 & 1.0221 & \\
\hline (c) Correct $N_{\text {pre }}$ & 2.341 & 94,482 & 40,359 & 134,841 & 0.2453 & 1.0000 & 134,847 \\
\hline
\end{tabular}

